\title{
Influence of the CSI 300 Stock Index Futures on the liquidity of the Stock Market of China-Analysis Based on the PSM and DID Method
}

\author{
Jinzhou Zhao ${ }^{1, a,{ }^{*}}$ and Yongbo Wang ${ }^{2, b}$ \\ ${ }^{1}$ Department of finance, South China University of Technology, Guangzhou, Guangdong, China \\ 2 Department of finance, South China University of Technology, Guangzhou, Guangdong, China \\ ajinzhou_zhao@126.com, b1028631490@qq.com
}

Keywords: CSI 300 stock index futures, liquidity, propensity score matching method, difference in difference estimation method.

\begin{abstract}
This paper used the data of Chinese A share market from 2009 to 2004.The propensity score matching method was used to match the index stocks with non-index stocks, then this two group of samples were studied with difference in difference model to explore the changes of liquidity of stock market since the launch of CSI 300 stock index futures. The results show that:the CSI 300 stock index futures trading generated capital crowding out effect on the spot market, which resulted in the significant reduction of both index stocks and non-index stocks' liquidity; due to the correlation with index futures in hedging, the index stocks suffered less influence than non-index stocks, and the liquidity gap between this two kinds of stocks showed an enlarging trend.
\end{abstract}

\section{沪深300股指期货对股票现货市场流动性的影响 一基于PSM方法和DID方法的分析}

\author{
赵锦洲 ${ }^{1, a^{*}}$, 王泳波 $2, \mathrm{~b}$, \\ 1华南理工大学金融系, 广州, 广东, 中国 \\ 2华南理工大学金融系, 广州, 广东, 中国 \\ a jinzhou_zhao@126.com, b1028631490@qq.com
}

关键词：沪深300股指期货；流动性；倾向得分匹配；双重差分法

中文摘要. 本文利用2009年至2014年的中国A股市场的数据, 首先通过倾向得分匹配法匹配出 与沪深300成分股相匹配的非成分股, 然后对这两组样本进行双重差分模型研究, 探讨沪深300 股指期货推出以来股票现货市场的流动性变化。研究结果表明: 沪深300股指期货的交易会对 现货市场产生资金挤出效应，现货市场的成分股和非成分股的流动性都有显著降低; 与股指 期货在套期保值方面的相关性使成分股的流动性受到的影响小于非成分股, 且成分股与非成 分股的流动性差距呈扩大趋势。

\section{1. 引言}

2010 年融资融券试点和股指期货的推出, 结束了中国证券市场只能做多不能做空的历史。 沪深 300 股指期货上市后发展非常迅猛, 其交易量在上市后三天内便超越了沪市 A 股的成交 量。但是, 与巨大的成交量相比, 股指期货的持仓量却远低于正常水平, 整个市场的成交持 
仓比非常高, 在期指推出初期成交持仓比甚至达到 30 左右, 市场投机气氛较重, 很可能会对 证券市场的流动性产生不良的影响。本文正是利用 PSM 方法和 DID 方法对我国 A 股市场的日 交易数据进行实证分析，研究沪深 300 股指期货的推出对我国股票现货市场流动性的影响。

\section{1 文献回顾}

部分学者研究表明, 股指期货的推出增强了股票现货市场的流动性。Kawaller 等（1987） ${ }^{[1]}$ 以标准普尔 500 指数及其成分股作为研究对象, 发现指数成分股的平均价格差额有明显降 低，指数期货的交易增强了股票现货市场的流动性。Chau 等（2007） ${ }^{[2]}$ 人从市场流动性和对 交易信息的反映水平的角度研究了全球股指期货对相应现货市场活力影响，结果表明股指期 货能够增强市场流动性。在国内, 丽金梁 (2012) ${ }^{[3]}$ 等人基于 EGARCH 模型参数估计的研究结 果认为, 沪深 300 指数期货的推出提升了股票市场的流动性和价格发现能力。

也有学者认为，股指期货的推出减弱了股票现货市场的流动性。Gould（1988） ${ }^{[4]}$ 对标准 普尔 500 指数期货合约和现货合约的套利机制进行研究, 发现当指数期货被不正确定价时, 期货市场和现货市场会发生套利现象，降低市场流动性。Duffie 等（1990） ${ }^{[5]}$ 认为，股指期 货市场巨大的交易量会耗尽现货市场的流动性来源, 并且导致股票现货市场的股票价格低于 其内在价值。在国内, 许红伟和吴冲锋 (2012) ${ }^{[6]}$ 利用联立方程模型研究沪深 300 股指期货 的影响, 认为股指期货推出的初期, 现货市场资金的增量小于转移到股指期货市场的资金量, 股票市场的换手率下降、流动性恶化。

同时也有研究指出，在证券市场的不同发展阶段，股指期货的会对股票现货市场产生不 同的影响。罗洎和王莹（2011） ${ }^{[7]}$ 认为在平稳运行的股指期货市场中，股指期货的推出都会 显著增加其市场流动性; 然而, 如果股指期货市场具有较强的投机性, 整个现货市场的流动 性反而会减弱。闻岳春和王泳（2012） ${ }^{[8]}$ 通过对沪深 300 股指期货推出后不同阶段股票日交 易量变化进行比较研究, 发现在股指期货推出初期, 市场的投机气氛较浓, 股指期货市场会 吸引部分高风险偏好投资者的资金，减少现货市场的资金流量，短时间内现货市场流动性减 弱; 在股指期货推出后半年内, 投资者对股指期货市场和现货市场的关系逐渐形成理性认识, 大批套利和套期保值者入市投资，两个市场的流动性有较大提高；而从长远分析，股市流动 性会最终保持在股指期货推出前的状态，股指期货的交易并不会实际影响现货市场的流动性。

显然，目前学者关于股指期货的推出对股票现货市场流动性的影响大致有三种观点：股 指期货的推出增强了现货市场的流动性、降低了现货市场的流动性或者在不同阶段有不同的 影响。那到底沪深 300 股指期货的推出对中国股票现货市场的流动性有什么影响? 本文正是 从实证的角度研究、回答这个问题。

\section{2 股指期货对股票现货市场流动性影响的理论分析}

1、对股票现货市场流动性的整体影响

股指期货主要从两个方面影响股票现货市场的流动性，分别是其对股票现货市场的资金 挤出效应和增量资金效应。挤出效应会减弱股票现货市场的流动性，增量资金效应则会提升 市场的整体流动性，股指期货的实际影响是这两个效应的综合结果。

资金挤出效应又称交易转移效应。作为两种金融产品，股指期货和股票现货之间有一定 的替代关系，然而股指期货在避险、套利等方面的独特优势会吸引大部分市场资金流向股指 期货市场，减少股票现货市场的资金流量和交易量，降低现货市场的流动性。

增量资金效应。股指期货的推出，打破了证券市场原先的交易结构和机制，将做空机制 引进了市场，完善了整个市场的避险和套利机制。市场结构和市场机制的优化，给投资者带 来了更多的获利机会和更稳健的投资方式。对于套利和套期保值者而言，股指期货的推出让 其发现了更多的投资机会和套利机会，会吸引增量资金进入到证券市场。而对于风险厌恶者 而言，股指期货的推出无疑降低了期现市场的投资风险，提高了收益的稳定性，同样会促使 更多投资者将资金投入到证券市场。 
2、对指数成分股和非指数成分股流动性的不同影响

股指期货的推出对指数成分股和非指数成分股的影响也是不同的，也需要从挤出效应和 增量效应两个方面综合分析。

一方面，股指期货的推出会吸引现货市场的部分资金流向股指期货市场，现货市场的指 数成分股和非指数成分股的流动性都会因此受到消极影响, 现货流动性有降低的可能, 而且 由于指数成分股和股指期货的替代性强于非指数成分股和股指期货的替代性，指数成分股的 挤出效应会更大。

另一方面，股指期货的推出会带动更多的资金流入到期现市场中，给股票现货市场的流 动性带来积极影响。而且投资者在进行投资结构安排的时候，通常会利用股指期货和指数成 分股的搭配达到避险或套利的目的，这样会分流部分资金到指数成分股交易中，从而给成分 股流动性带来积极影响。

总之，要分析股指期货的推出对成分股和非成分股的实际总影响必须看以上两种效应的 综合作用结果。

\section{2. 实证模型的构建及变量选取}

为了更好地研究沪深 300 股指期货的推出对股票现货市场流动性的影响，本文将倾向得 分匹配法（PSM）与双重差分法（DID）相结合。

\section{1 变量选取}

在 PSM 模型和 DID 模型中，用到以下变量（以下变量中下标 “i”表示第 1 阶段到第 4 阶段的其中一个阶段 i):

1、变量 treat

样本股在 2010 年 4 月 16 日到 2014 年 3 月 31 日是否为沪深 300 成分股, 用变量 treat 表 示, treat $=1$ 表示股票在该时段始终属于沪深 300 成分股, treat $=0$ 表示股票在该时段从未属于 成分股。

2、变量 $\operatorname{lnill}_{i}$

本文采用 Amihud 非流动性指标的自然对数衡量股票的流动性，简称非流动性指标，该 指标反映的是单位时间内单位交易量对价格的冲击, 用 $\operatorname{lnill}_{\mathrm{i}}$ 表示。与一般流动性衡量指标相 反，在分析中， nnill $_{\mathrm{i}}$ 越大说明该股票流动性越差。具体计算公式如下:

$$
\ln i l_{i}=\ln \left(\sum_{t=1}^{n_{i}} \frac{\left(H_{i, t}-L_{i, t}\right) /\left(V_{i, t} \times O_{i, t}\right)}{n_{i}}\right)
$$

其中, $\mathrm{n}_{\mathrm{i}}$ 表示阶段 $\mathrm{i}$ 的交易日天数。 $\mathrm{O}_{\mathrm{i}, \mathrm{t}}, \mathrm{H}_{\mathrm{i}, \mathrm{t}}$ 和 $\mathrm{L}_{\mathrm{i}, \mathrm{t}}$ 表示第 $\mathrm{i}$ 阶段的第 $\mathrm{t}$ 个交易日的开盘价, 最高价和最低价， $\mathrm{V}_{\mathrm{i}, \mathrm{t}}$ 表示第 $\mathrm{i}$ 阶段的 $\mathrm{t}$ 个交易日的成交量。

3、变量 $\operatorname{lnstd}_{\mathrm{i}}$

股票收益标准差（股票收益率的波动率）的自然对数，用 $\operatorname{lnstd}_{\mathrm{i}}$ 表示，该指标衡量的是股 票在该阶段的波动程度。股票收益率 $\mathrm{r}_{\mathrm{t}}$ 由公式 $\mathrm{r}_{\mathrm{t}}=\ln \left(\mathrm{P}_{\mathrm{t}}\right)-\ln \left(\mathrm{P}_{\mathrm{t}-1}\right)$ 计算得到, 其中 $\ln \left(\mathrm{P}_{\mathrm{t}}\right)$ 表示某 股票在第 $\mathrm{t}$ 天收盘价的自然对数。

4、变量 syriski $_{i}$

股票的系统风险，用该股票的 beta 值和市场收益标准差（股票市场收益用中证 800 指数 计算得到）的乘积计算得到, 用 syrisk $_{i}$ 表示。该指标衡量的是股票在该阶段的系统性风险。

5、变量 $\operatorname{lnsize} \mathrm{i}_{\mathrm{i}}$

股票规模的自然对数，用 $\operatorname{lnsize}_{\mathrm{i}}$ 表示，该指标衡量的是股票在该阶段的规模大小。用股 票在该阶段 $\mathrm{A}$ 股的平均规模（该股平均流通 $\mathrm{A}$ 股数*该股平均股价）表示。该阶段平均 $\mathrm{A}$ 流通 股数用期初该股票 A 股规模和期末该股票 A 股规模的平均值来衡量。 


\section{2 变量选取构建倾向得分模型}

沪深 300 指数成分股和非成分股的倾向得分匹配是本文实证分析中的关键步骤。将倾向 得分匹配法引入到研究中, 对成分股和非成分股进行匹配, 不仅可以在一定程度上控制内生 性问题, 还可以保证研究的样本量, 从而提高研究结果的可信度（杨阳和万迪昉，2010） ${ }^{[9]}$ 。 在此处, 将构建倾向得分模型, 利用 Logit 模型对模型变量进行回归, 估计成分股和非成分 股的倾向得分。

\section{3 构建双重差分模型}

本文中，双重差分模型将与倾向得分模型配合使用。二者配合使用，可以将时间差异和 组间差异进行比较, 消除某些不可观测因素对研究结果的影响。双重差分模型的回归中, 一 共有 618 支股票，其中 194 支是沪深 300 成分股 (用符号 CSI 表示)，而其余 424 支是利用倾 向得分的核匹配法匹配出来的非成分股 (用符号 NCSI 表示)。本文中, 将 lnill 作为衡量股票 流动性的指标，该指标数值越大，表明股票流动性越差。构建的双重差分模型为:

$$
\ln i l l=\mathrm{c}_{1}+\mathrm{a}_{1} \text { treat }+\sum_{\mathrm{j}=1}^{3} \mathrm{a}_{2, \mathrm{j}} \text { stg }_{j+1}+\sum_{\mathrm{j}=1}^{3} \mathrm{a}_{3, \mathrm{j}} \text { treat }^{*} \text { stg }{ }_{j+1}+\mathrm{a}_{4} \text { syrisk }+\mathrm{a}_{5} \ln s t d+\mathrm{a}_{6} \ln s i z e+v
$$

上述模型中, 常数项表示非成股第 1 阶段的流动性。其中, $\operatorname{stg}_{2}, \operatorname{stg}_{3}$ 和 $\operatorname{stg}_{4}$ 是三个二值 变量, 如果是第 $\mathrm{i}$ 阶段的数据, 则 $\mathrm{stg}_{\mathrm{i}}=1$, 其他三个为零, $\mathrm{stg}_{2}$ 的系数用于衡量非成分股第 2 阶段和第 1 阶段的流动性差异。交互项 $\operatorname{treat}^{*} \operatorname{stg}_{2}$, treat* $\operatorname{stg}_{3}$ 和 $\operatorname{treat}^{*} \mathrm{stg}_{4}$ 是双重差分估计量, treat* ${ }^{*} \operatorname{tg}_{\mathrm{i}}$ 分别表示第 $\mathrm{i}$ 阶段沪深 300 成分股与非成分股流动性差异的变化。 $\mathrm{v}$ 表示误差项。其 他变量的含义和上一部分介绍的一致。

\section{3. 实证检验}

\section{1 数据来源及处理}

1、数据来源

本文的所有股票数据均来自上海恒生聚源有限公司的金融数据库。选取我国证券市场中 的沪深 300 股指期货, 沪深 300 成分股和 $\mathrm{A}$ 股市场其他股票作为研究对象, 以沪深 300 成分 股代表成分股现货市场，以非沪深 300 成分股代表非成分股现货市场。研究区间为 2009 年 1 月 1 日到 2014 年 03 月 31 日，由于这一期间能够覆盖沪深 300 股指期货推出前后的时间段， 且推出后市场的几个典型表现也包含在其中。

2、数据处理

为了更好地分析股指期货对股票现货市场流动性的影响，进行了如下数据处理：

(1) 将样本区间分成 4 个阶段。在综合考虑了股指期货发展阶段中的各项重大政策和股 票现货市场投资气氛后，将样本区间细分成 4 个阶段。

沪深 300 股指期货 2010 年 4 月 16 日上市交易初期交易过于活跃，成交持仓比居高不下， 学界认为此阶段 “投机味” 较浓。2010 年六月中旬起, 监管当局着力整治股指期货交易市场, 同时套期保值者开始入市，于是从 8 月份起，市场逐步趋于稳定并保持稳定到 2013 年 6 月份。 2013 年 6 月到 2014 年 3 月, 成交持仓比有较大的波动。根据上述分析, 将研究阶段分为以 下 4 个阶段： 2009 年 1 月 1 日到 2010 年 4 月 15 日为股指期货推出前的阶段，也即研究基准 对照阶段。2010 年 4 月 16 日到 2010 年 7 月 15 日为投机味较浓的阶段作为第 2 阶段。 2010 年 7 月 16 日到 2013 年 6 月 15 日作为第 3 阶段, 股指期货市场较为稳定, 定价效率也较高。 2013 年 6 月 16 日到 2014 年 3 月 31 日股指期货成交持仓比变化较大，作为第 4 阶段。

(2) 提取成分股。

(3) 提取非成分股。

（4）补充缺失交易日的股票数据。 
（5）计算研究中用到的相关指标。

\section{2 倾向得分匹配法结果}

倾向得分模型的结果如表 1 所示：在 $1 \%$ 的显著性水平下， syrisk $1 、 \operatorname{lnstd}_{1} 、 \operatorname{lnill}_{1}$ 和 $\operatorname{lnsize}_{1}$ 的系数都都显著不为零。模型的卡方检验统计量对应的 $\mathrm{p}$ 值为 0 , 模型的虚拟判定系数 Pseudo $R^{2}$ 的值为 0.5921 , 说明模型的拟合效果较好, 对估计的倾向得分值的解释能力较强, 可以很 好平衡对比样本的混杂因素。倾向得分是样本固有属性的合成代表, 通过比较倾向得分可以 判断干预组和控制组是否有足够的重叠 (公共支撑域), 公共支撑域的大小决定了干预组和对 比组之间是否有可比性，在该结果中，倾向得分的公共支撑域为 [0.01472273，0.99997496], 较大的公共支撑域降低了倾向得分匹配法对具体匹配方法的敏感度，提高了倾向得分的匹配 效果，保证了匹配的有效性。

利用上述倾向得分模型计算出的倾向得分，将成分股和非成分股进行倾向得分匹配。本 文中用到的匹配方法是核匹配法，使用的是 STATA 软件的 attk.ado 程序。经过核匹配，有 424 个非成分股与先前挑选出的 194 个沪深 300 成分股相匹配。

表1 倾向得分估计结果

\begin{tabular}{|c|c|c|c|c|c|}
\hline treat & 系数 & 标准误差 & t统计量 & $\mathrm{P}>|\mathrm{t}|$ & $95 \%$ 置信区间 \\
\hline syrisk $_{1}$ & 108.509 & 30.327 & -3.58 & $0.000 * *$ & {$[167.948,49.069]$} \\
\hline lnstd $_{1}$ & 2.057 & 0.276 & 7.46 & $0.000 * *$ & {$[1.516,2.597]$} \\
\hline lnil1 $_{1}$ & -2.789 & 0.249 & -11.20 & $0.000 * *$ & {$[-3.277,-2.301]$} \\
\hline lnsize $_{1}$ & 0.932 & 0.136 & 6.88 & $0.000 * *$ & {$[0.667,1.198]$} \\
\hline $\mathrm{c}$ & -48.544 & 3.517 & -13.80 & $0.000 * *$ & {$[-55.437,-41.651$} \\
\hline \multicolumn{5}{|c|}{ Pseudo $\mathrm{R}^{2}=0.5921$} & \multicolumn{4}{|c|}{ obs $=1199$} & Prob $>$ chi2 $=0.0000$ \\
\hline
\end{tabular}

\section{3 双重差分法实证结果}

表 2 给出了基于式 (1) 的双重差分模型的回归结果。控制变量有三个, 分别是系统性风 险 syrisk, 股票波动性 lnstd 和股票规模 Insize。 $\mathrm{T}_{\mathrm{i}}(\mathrm{i}=1 ， 2 ， 3 ， 4 ）$ 表示数据所衡量的阶段, CSI 表示沪深 300 成分股, NCSI 表示非沪深 300 成分股。

常数项表示第 1 阶段非成分股的流动性，其估计值显著小于 0 。代表控制组和实验组的 虚拟变量 treat 代表在第 1 阶段成分股和非成分股的流动性差异, 其估计值显著为负, 说明第 1 阶段成分股的流动性显著高于非成分股。系统风险 syrisk 的系数显著为正, 说明股票的系统 性风险越大, 其流动性越小。波动率 $\operatorname{lnstd}$ 的系数显著为正, 表明股票波动率越大, 其流动性 越小。规模 $\operatorname{lnsize}$ 的系数显著为正, 表明股票规模越大，股票流动性越小，这与衡量流动性 的 lnill 指标构建有关，该指标分母的其中一项为成交量，规模大的股票往往具有较大的成交 量, 因而规模越大的股票 Inill 可能越大, 以该指标衡量的流动性就会越小。时间虚拟变量 $\operatorname{stg}_{2}$, $\operatorname{stg}_{3}$ 和 $\operatorname{stg}_{4}$ 表示非成分股流动性变化的时间趋势，其系数都显著为正，表明沪深 300 股指期货 推出以后，股指期货对股票现货市场的交易资金转移效应大于其给整个证券的市场带来的增 量资金效应，证券市场的增量资金及部分原先在非成分股交易中使用的资金转移到期货市场 中, 非成分股的流动性反而降低了。双重差分估计量 treat*stg 表示与第 1 阶段相比, 成分股 与非成分股流动性差异的变化情况。 $\mathrm{treat}^{*} \mathrm{stg}_{2}$ 系数并不显著, 表明在股指期货推出初期, 成 分股与非成分股流动性差异并没有显著变化。 $\operatorname{treat}^{*} \mathrm{stg}_{3}$ 和 $\operatorname{treat}^{*} \mathrm{stg}$ 的系数显著为负，成分股 的流动性降低程度都显著小于非成分股, 说明与第 1 阶段相比, 成分股和非成分股的流动性 差异明显增大。

一方面，随着股指期货市场的发展完善，沪深 300 成分股和非成分股的流动性差异有扩 大趋势。因为随着期货市场的发展成熟、股指期货相关产品的推出以及投资者对期货市场认 
识的加深，投资者在构建投资组合的时候会将更多的资金投资到股指期货及相关产品中，部 分资金从现货市场流入到期货市场，当期货市场发展到一定程度以后，期现市场会逐渐趋向 一个相对稳定的关系，现货市场流动性变化幅度会减小。另一方面，沪深 300 成分股的流动 性受到的影响小于非成分股，因为成分股与股指期货在套期保值方面具有相关性，投资者利 用股指期货的交易进行套期保值或套利的时候，通常会利用成分股与股指期货形成投资组合， 所以股指期货交易的增加会给成分股带来部分流动性，而且这种影响随着股指期货市场的发 展完善越来越明显。

表2＼cjkstart双重差分法回归结果

\begin{tabular}{|c|c|c|c|c|c|}
\hline 变量 & 含义 & 系数 & 标准误差 & $\mathrm{t}$ 统计量 & p值 \\
\hline $\mathrm{c}_{1}$ & $\mathrm{~T}_{1}, \mathrm{NCSI}$ & -14.9763 & 0.3912 & -38.2809 & $0.00 * *$ \\
\hline treat & $\mathrm{T}_{1}$, CSI-NCSI & -1.3642 & 0.0944 & -14.4470 & $0.00 * *$ \\
\hline $\operatorname{stg}_{2}$ & $\mathrm{~T}_{2}-\mathrm{T}_{1}$, NCSI & 0.3867 & 0.0751 & 5.1500 & $0.00 * *$ \\
\hline $\operatorname{stg}_{3}$ & $\mathrm{~T}_{3}-\mathrm{T}_{1}, \mathrm{NCSI}$ & 0.8245 & 0.0766 & 10.7627 & $0.00 * *$ \\
\hline $\operatorname{stg}_{4}$ & $\mathrm{~T}_{4}-\mathrm{T}_{1}, \mathrm{NCSI}$ & 0.6612 & 0.0765 & 8.6456 & $0.00 * *$ \\
\hline treat* $\operatorname{stg}_{2}$ & $\mathrm{~T}_{2}-\mathrm{T}_{1}$, CSI-NCSI & 0.0352 & 0.1288 & 0.2730 & 0.78 \\
\hline treat ${ }^{*} \operatorname{stg}_{3}$ & $\mathrm{~T}_{3}-\mathrm{T}_{1}$, CSI-NCSI & -0.4055 & 0.1291 & -3.1416 & $0.00 * *$ \\
\hline treat ${ }^{*} \operatorname{stg}_{4}$ & $\mathrm{~T}_{4}-\mathrm{T}_{1}$, CSI-NCSI & -0.3219 & 0.1289 & -2.4970 & $0.01 * *$ \\
\hline syrisk & & 13.2355 & 9.1766 & 1.4423 & 0.15 \\
\hline lnstd & & 1.6952 & 0.0870 & 19.4771 & $0.00 * *$ \\
\hline Insize & & 0.5378 & 0.0161 & 33.4287 & $0.00 * *$ \\
\hline
\end{tabular}

表 3 是对控制相关因素后的 $\ln i 11$ 的差分和双重差分估计量进行检验的结果。以下对股 指期货推出以后现货市场中的成分股和非成分股流动性情况分别进行分析。股指期货推出的 初期，成分股流动性显著降低（0.4218），非成分股的流动性也显著降低（0.3867），两者流 动性差异变化不显著 (0.0352), 说明在股指期货推出的初期, 由于股指期货市场中大量投机 者的存在，期货市场中存在大量的投机性买卖，受到交易资金转移效应的影响，部分资金从 现货市场转移到期货市场，现货市场的流动性显著降低。在第 3 阶段，成分股和非成分股的 流动性也有显著降低 (分别是 0.4190 和 0.8245 ), 成分股的流动性与初期相比并没有很大变 化, 而非成分股的流动性有了较大的降低, 表明在第 3 阶段大量套期保值者入市后, 市场中 的投机性交易减少，市场处于稳定发展的时期，此时市场趋于理性，资金在期现市场中的分 配以及在成分股和非成分股之间的分配也会趋于稳定。在第 4 阶段, 与市场初期相比, 成分 股的流动性显著降低，非成分股也显著降低，但其降低程度小于第 3 阶段，表明此阶段期货 市场较为成熟，且比较活跃，成交持仓比较大，整个证券市场的活跃增加了现货市场的资金 流，因此现货市场的流动性优于第 3 阶段。总而言之，股指期货推出以后，股票现货市场的 流动性有显著降低。 
表3 控制相关因素后的差分和双重差分估计量检验

\begin{tabular}{|c|c|c|c|}
\hline 变量 & 差异 & F检验统计量 & $\mathrm{p}$ 统计量 \\
\hline $\mathrm{T}_{2}-\mathrm{T}_{1}$, CSI & 0.4218 & 15.1978 & $0.00^{* *}$ \\
\hline $\mathrm{T}_{2}-\mathrm{T}_{1}, \mathrm{NCSI}$ & 0.3867 & 26.5229 & $0.00^{* *}$ \\
\hline $\mathrm{T}_{2}-\mathrm{T}_{1}$, CSI-NCSI & 0.0352 & 0.0745 & 0.78 \\
\hline $\mathrm{T}_{3}-\mathrm{T}_{1}$, CSI & 0.4190 & 14.3720 & $0.00^{* *}$ \\
\hline $\mathrm{T}_{3}-\mathrm{T}_{1}, \mathrm{NCSI}$ & 0.8245 & 115.8365 & $0.00^{* *}$ \\
\hline $\mathrm{T}_{3}-\mathrm{T}_{1}$, CSI-NCSI & -0.4055 & 9.8697 & $0.00^{* *}$ \\
\hline $\mathrm{T}_{4}-\mathrm{T}_{1}, \mathrm{CSI}$ & 0.3393 & 9.3209 & $0.00^{* *}$ \\
\hline $\mathrm{T}_{4}-\mathrm{T}_{1}, \mathrm{NCSI}$ & 0.6612 & 74.7467 & $0.00^{* *}$ \\
\hline $\mathrm{T}_{4}-\mathrm{T}_{1}, \mathrm{CSI}-\mathrm{NCSI}$ & -0.3219 & 6.2349 & $0.01^{* *}$ \\
\hline
\end{tabular}

值得注意的是，股指期货还存在着吸引场外增量资金入市的效应，会给包括股票现货市 场在内的整个证券市场的流动性带来积极的影响。在本文的研究中，增量资金效应的对现货 市场流动性影响要远小于股指期货给现货市场带来的资金挤出效应的影响，因此实证结果显 示股票现货市场的流动性有显著降低。

\section{4. 结论}

本文将倾向得分匹配法和双重差分法相结合，研究股指期货的推出对股票现货市场流动 性的影响。文中以非流动性指标作为衡量股票流动性的指标, 将样本区间分成 4 个阶段进行 分析, 得到了以下结论:

股指期货推出后的每个阶段，沪深 300 成分股和非成分股，流动性都有显著降低。说明 股指期货将做空机制引入证券市场后，其本身具有套期保值和投机的功能会吸引大量的资金 流入到股指期货市场。而且，伴随股指期货的推出而催生的与期指相关的金融产品也会吸引 部分的资金，从而降低现货市场的流动性。

股指期货推出后，非成分股流动性受到的影响大于成分股，而且这种差异在后来的阶段 呈逐步扩大的趋势。这表明沪深 300 股指期货的推出虽然对成分股和非成分股都会产生资金 挤出效应，但是套期保值者入市后，股指期货相关交易的增加也会给成分股带来部分流动性， 所以成分股流动性降低程度低于非成分股。同时，由于成分股和股指期货在套期保值、套利 的功能上具有相关性，股指期货交易给成分股带来的部分流动性也会使成分股流动性受到的 影响并没有非成分股那么大，所以成分股和非成分股流动性的差异会扩大。

\section{References}

[1] Kawaller ,K L, P D Koch, and T W Koch,1987. The Temporal Price Relationship between S\&P500 Futures and S\&P 500 Index,Journal of Finance,Vol.42,No.1:1309-1329.

[2] Chau , F,Holmes,and Paudyalk,2007. The Impact of Universal Stock Futures on Feedback Trading and Volatility Dynamics,Journal of Business Finance \& Accounting Vol.35, No.1:227-249.

[3] LI Jinliang,LEI Yao,LI Shujingi. Market depth, liquidity and Volatility -- the impact of Shanghai and Shenzhen 300 stock index futures on the spot market [J]. Journal of Financial Research,,2012(06).

[4] Gould, F J,1988. Stock Index Futures: the Arbitrage Cycle and Portfolio Insurance, Financial Analysts Journal,48-62. 
[5] Kupiec ,G P, and P A White,1990. A Primer on Program Trading and Stock Price Volatility:A Survey of the Issues and the Evidence, Working Paper.

[6] Xu Hongwei.Wu Chongfeng. Is the CSI 300 stock index futures improves the aualitv of China's stock market? -- An Empirical Study Based on simultaneous equation model [J]. Nankai Business Review, 2012(4).

[7] Luo Mian, Wang Ying. The impact of stock index futures on volatility and liquidity of securities market -- Empirical Study Based on Chinese market [J]. Macroeconomics, 2011(6).

[8] Wen Yuechun, Wang Yong. The impact of stock index futures on stock market liquidity [J]. Financial Theory \& Practice,,2012(7).

[9] YANG Yang WAN Difang. Can stock index futures really stabilize the market? [J]. Journal of Financial Research,, 2010(12). 